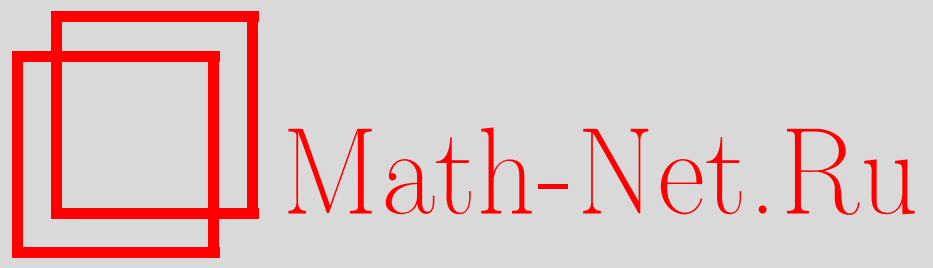

Обцероссийский математический портал

С. Л. Лесик, Аттрактор неавтономного гиперболического уравнения с малым параметром, Матем. заметки, 2000, том 67, выпуск 2, 308-311

DOI: https://doi.org/10.4213/mzm841

Использование Общероссийского математического портала Math-Net.Ru подразумевает, что вы прочитали и согласны с пользовательским соглашением http://www.mathnet.ru/rus/agreement

Параметры загрузки:

IP : 54.164 .48 .24

26 апреля 2023 г., 03:07:20 







\section{АТТРАКТОР НЕАВТОНОМНОГО ГИПЕРБОЛИЧЕСКОГО УРАВНЕНИЯ С МАЛЫМ ПАРАМЕТРОМ}

\section{С. Л. Лесик}

В ограниченной области $\Omega \subset \mathbb{R}^{n}$ рассматривается гиперболическое уравнение вида

$$
\varepsilon \partial_{t}^{2} u+\gamma(t) \partial_{t} u-\Delta u+f(u, t)+\varphi(x, t)=0
$$

при граничном условии Дирихле

$$
\left.u\right|_{\partial \Omega}=0 .
$$

Здесь $\varepsilon \in\left(0 ; \varepsilon_{0}\right]$ - малый параметр; функции $\gamma(t), f(u, t), \varphi(x, t)$ являются трансляционно-компактными функциями (см [1]) в пространствах, определяемых ниже.

Полагая мальй параметр $\varepsilon$ в уравнении (1) равным нулю, получаем параболическое уравнение вида

$$
\gamma(t) \partial_{t} u-\Delta u+f(u, t)+\varphi(x, t)=0 .
$$

В автономном случае уравнения типа (1) и (3) изучались в [2]-[4].

Введем некоторые обозначения.

ОПРЕДЕЛЕниЕ 1 . Обозначим через $\Xi_{1}$ пространство $H^{1, l o c}(\mathbb{R})$. Топология в пространстве $\Xi_{1}$ определяется следующим образом: последовательность $\left\{\gamma_{n}(t)\right\}_{n \in \mathbb{N}}$ сходится к $\gamma(t)$ в $\Xi_{1}$, если для любого интервала $\left[t_{1}, t_{2}\right]$

$$
\left\|\gamma_{n}(s)-\gamma(s)\right\|_{H^{1}\left[t_{1}, t_{2}\right]}^{2} d s \rightarrow 0
$$

при $n \rightarrow \infty$.

ОПРЕДЕЛЕНиЕ 2 . Обозначим через $\Xi_{2}$ пространство $C^{1, \operatorname{loc}_{(}}(\mathbb{R} \times \mathbb{R}, \mathbb{R})$. Топология в пространстве $\Xi_{2}$ задается следуюшим образом: последовательность $\left\{f_{n}(u, t)\right\}_{n \in \mathbb{N}}$ сходится к $f(u, t)$ в $\Xi_{2}$, если для любого компакта $K \in \mathbb{R} \times \mathbb{R}$ имеет место сходимость $\left\|f_{n}(u, t)-f(u, t)\right\|_{C^{1}(K)} \rightarrow 0$ при $n \rightarrow \infty$.

ОПРЕДЕлЕниЕ 3 . Обозначим через $\Xi_{3}$ пространство $W_{2}^{1, l o c}\left(\mathbb{R}, L_{2}(\Omega)\right)$. Последовательность $\left\{\varphi_{n}(x, t)\right\}_{n \in \mathbb{N}}$ сходится к $\varphi(x, t)$ в $\Xi_{3}$, если для любого интервала $\left[t_{1}, t_{2}\right]$ имеет место сходимость

$$
\int_{t_{1}}^{t_{2}}\left\|\varphi_{n}(x, s)-\varphi(x, s)\right\|^{2} d s \rightarrow 0, \quad \int_{t_{1}}^{t_{2}}\left\|\partial_{t} \varphi_{n}(x, s)-\partial_{t} \varphi(x, s)\right\|^{2} d s \rightarrow 0
$$

при $n \rightarrow \infty$. 
ОПРЕДЕЛЕНИЕ 4. Функция $\gamma(t)$, принадлежащая пространству $\Xi_{1}$, называется транслячионно компактной в $\Xi_{1}$, если ее оболочка

$$
\mathcal{H}_{1}(\gamma)=[T(t) \gamma(s) \mid t \in \mathbb{R}]_{\Xi_{1}}
$$

компактна в $\Xi_{1}$, где [ $] \Xi_{1}$ - замыкание в пространстве $\Xi_{1}, T(t) \gamma(s)=\gamma(s+t)($ см. [5], [6]).

Трансляционная компактность функций $f \in \Xi_{2}$ и $\varphi \in \Xi_{3}$, а также их оболочек $\mathcal{H}_{2}(f)$ и $\mathcal{H}_{3}(\varphi)$ определяются аналогично.

Предполагается, что функции $\gamma(t), f(u, t), \varphi(x, t)$ обладают следующими свойствами:

i) $\gamma(t)$ - трансляционно компактна в $\Xi_{1}, 0<\gamma_{0} \leqslant \gamma(t) \leqslant \Gamma \forall t \in \mathbb{R}$ и $\dot{\gamma}(t)=\partial \gamma(t) / \partial t$ такова, что $|\dot{\gamma}(t)| \leqslant C$, где $C$ некоторая положительная константа;

ii) функция $\varphi(x, t)$ - трансляционно-компактна в пространстве $\Xi_{3}$;

iii) функция $f(u, t)$ трансляционно-компактна в $\Xi_{2}$ и удовлетворяет следующим условиям:

$$
\begin{gathered}
f(u, t) \cdot u \geqslant-C-\left(\lambda_{1}-\delta_{1}\right) u^{2}, \quad\left|f_{u}^{\prime}(u, t)\right| \leqslant \mathrm{C}(1+|u|)^{p}, \\
\left|f_{t}^{\prime}(u, t)\right| \leqslant \mathrm{C}(1+|u|)^{(p+1)}
\end{gathered}
$$

где $p<2, t \in \mathbb{R}, C>0$; здесь $\lambda_{1}-$ первое собственное число оператора $-\Delta$ с однородным условием Дирихле на границе;

iv) функция $F(u, t)=\int_{0}^{u} f(v, t) d v$ удовлетворяет неравенствам:

$$
\text { 1) } F(u, t) \geqslant-\frac{1}{2}\left(\lambda_{1}-\delta_{1}\right) u^{2}-\frac{1}{2} C_{1} \text {, }
$$

где $C_{1}, \delta_{1}$ - положительные константы;

$$
\text { 2) } \alpha f(u, t) u \geqslant \delta_{2} F(u, t)+F_{t}^{\prime}(u, t)-C \text {, }
$$

а константы $\delta_{2}$ и $\alpha$ таковы, что

$$
\alpha=\frac{\gamma_{0} \lambda_{1}}{2 \Gamma^{2}}, \quad \delta_{2}<\gamma_{0} \min \left(\frac{1}{\Gamma^{2}} ; \frac{1}{\varepsilon_{0}}\right)
$$

Обозначим через $\Xi$ пространство $\left(\Xi_{1} \times \Xi_{2} \times \Xi_{3}\right)$. Вектор $\sigma(t)=(\gamma(t), f(u, t), \varphi(x, t))$ назовем символом уравнения (1). Рассмотрим оболочку функции $\sigma(t)$ в $\Xi$

$$
\mathcal{H}(\sigma)=[T(t) \sigma(s) \mid t \in \mathbb{R}]_{\Xi},
$$

где [·] $\Xi$ - замыкание в пространстве $\Xi, T(t) \sigma(s)=\sigma(s+t)$. Множество $\mathcal{H}(\sigma)$ назовем оболочкой символа $\sigma$. Так как $\mathcal{H}(\sigma) \subset \mathcal{H}_{1}(\gamma) \times \mathcal{H}_{2}(f) \times \mathcal{H}_{3}(\varphi)$, оболочка $\mathcal{H}(\sigma)$ компактна в $\Xi$.

Следуя [6], далее будем рассматривать семейство уравнений следующего вида:

$$
\varepsilon \partial_{t}^{2} u+\beta(t) \partial_{t} u-\Delta u+g(u, t)+\psi(x, t)=0
$$

при граничном условии Дирихле

$$
\left.u\right|_{\partial \Omega}=0,
$$

где символ $\rho(t)=(\beta(t), g(u, t), \psi(x, t))$ уравнения (4) принадлежит оболочке $\mathcal{H}(\sigma)$.

Компоненты любого символа $\rho$ из оболочки $\mathcal{H}(\sigma)$ удовлетворяет тем же условиям i)-iv) что и компоненты символа $\sigma$. 
Теорема 1. Пусть компоненты символа $\sigma=(\gamma(t), f(u, t), \varphi(x, t))$ удовлетворяют условиям i)-iv) и начальные условия

$$
\left.u\right|_{t=\tau}=u_{\tau} ;\left.\quad \frac{\partial u}{\partial t}\right|_{t=\tau}=p_{\tau}
$$

таковь, что $u_{\tau} \in H_{0}^{1}(\Omega)$ и $p_{\tau} \in L_{2}(\Omega)$. Тогда задача (4)-(6) однозначно разрешима в классе $C\left([\tau ; T], H_{0}^{1}(\Omega) \times L_{2}(\Omega)\right) \quad \forall T>\tau$ для любого $\rho(t) \in \mathcal{H}(\sigma)$.

Доказательство этой теоремы можно произвести методом, предложенньм в [7].

Таким образом, корректно определен оператор $\mathcal{U}_{\rho}^{\varepsilon}(t, \tau)$ сдвига по траекториям решения задачи (4)-(6). Оператор $\mathcal{U}_{\rho}^{\varepsilon}(t, \tau), t \geqslant \tau, \tau \in \mathbb{R}, \rho \in \mathcal{H}(\sigma), 0<\varepsilon \leqslant \varepsilon_{0}$, действует в пространстве $H_{0}^{1}(\Omega) \times L_{2}(\Omega)$ по формуле

$$
\mathcal{U}_{\rho}^{\varepsilon}(t, \tau)\left(u(\tau), \frac{\partial u_{\rho}^{\varepsilon}}{\partial t}(\tau)\right)=\left(u_{\rho}^{\varepsilon}(t), \frac{\partial u_{\rho}^{\varepsilon}}{\partial t}(t)\right)
$$

Кроме того, вьполняется трансляционное тождество

$$
\mathcal{U}_{\rho}^{\varepsilon}(t+s, \tau+s)=\mathcal{U}_{T(s) \rho}^{\varepsilon}(t, \tau)
$$

для всякого $s \geqslant 0$.

Построим оператор $S_{t}(\varepsilon)$, по следующей формуле:

$$
S_{t}(\varepsilon)\left(u_{0}, p_{0}, \rho\right)=\left(u_{\rho}^{\varepsilon}(t), \frac{\partial u_{\rho}^{\varepsilon}}{\partial t}(t), T(t) \rho\right),
$$

где $u_{\rho}^{\varepsilon}(t)$ - решение задачи (4)-(6) с символом $\rho$ и $\tau=0$.

Нетрудно проверить, что таким образом определенный оператор $S_{t}(\varepsilon)$ является полугруппой, действующей из пространства $(E \times \Xi)$ в $(E \times \Xi)$, где $E=H_{0}^{1}(\Omega) \times L_{2}(\Omega)$.

ТЕОРема 2. При выполнении условий i)-iv) полугруппа $S_{t}(\varepsilon)$, определенная выше, для всякого $\varepsilon, \varepsilon_{0} \geqslant \varepsilon>0$, обладает максимальным компактным $в(E \times \Xi)$ аттрактором $\mathcal{A}(\varepsilon)$. Кроме того, семейство аттракторов $\mathcal{A}(\varepsilon)$ равномерно по $\varepsilon_{0} \geqslant \varepsilon>0$ ограничено в $\left(H^{2}(\Omega) \cap H_{0}^{1}(\Omega) \times H_{0}^{1}(\Omega) \times \Xi\right)$.

Рассмотрим семейство уравнений (4) при $\varepsilon=0$ :

$$
\beta(t) \partial_{t} u-\Delta u+g(u, t)+\psi(x, t)=0
$$

при граничном условии Дирихле

$$
\left.u\right|_{\partial \Omega}=0
$$

и начальном условии

$$
\left.u\right|_{t=\tau}=u_{\tau}
$$

где $u_{\tau} \in H_{0}^{1}(\Omega)$, символ $\rho(t)=(\beta(t), g(u, t), \psi(x, t))$ принадлежит оболочке $\mathcal{H}(\sigma)$. Аналогично автономному случаю (см. [2]) для задачи (7)-(9) доказываются теорема о существовании решения. По процессу $\mathcal{U}_{\rho}$, соответствующему оператору сдвига по траекториям решений задачи $(7),(8)$, строится полугруппа $S_{t}$, действующая в пространстве $H_{0}^{1}(\Omega) \times \Xi$.

ТЕорема 3. При выполнении условий i)-iv) полугруппа $S_{t}$ обладает аттрактором $\mathcal{A}$, компактным в $H^{2}(\Omega) \cap H_{0}^{1} \times \Xi$.

Определим множество $\mathcal{A}(0)$ следующим образом:

$$
\begin{aligned}
\mathcal{A}(0)= & \{(u(0), p(0), \rho(\cdot)) \mid u(t)-\text { решение задачи }(7),(8), \text { лежашее } \\
& \text { на аттракторе } \left.\mathcal{A} \text {, т.е. }(u(0), \rho) \in \mathcal{A} ; \quad p(t)=\frac{1}{\beta(t)}(\Delta u-g(u, t)-\psi)\right\} .
\end{aligned}
$$

Тогда из теоремы 3 следует, что $\mathcal{A}(0)$ компактно в $H_{0}^{1} \times L_{2}(\Omega) \times \Xi$. 
Tеорема 4. Семейство аттракторов $\mathcal{A}(\varepsilon), \varepsilon \in\left[0 ; \varepsilon_{0}\right]$, полунепрерывно сверху в точке $\varepsilon=0$ :

$$
\operatorname{dist}_{\left(E_{1} \times \Xi\right)}(\mathcal{A}(\varepsilon), \mathcal{A}(0)) \rightarrow 0 \quad n p u \quad \varepsilon \rightarrow 0,
$$

әде через $E_{1}$ обозначено пространство $H^{2}(\Omega) \cap H_{0}^{1}(\Omega) \times H_{0}^{1}(\Omega)$. Здесь

$$
\operatorname{dist}_{\left(E_{1} \times \Xi\right)}(A, B)=\sup _{a \in A} \inf _{b \in B}|a-b|_{\left(E_{1} \times \Xi\right)} .
$$

ОПРЕДЕЛЕНиЕ 5 . Обозначим через $\Pi_{1}$ оператор проецирования $E_{1} \times \Xi$ на первую компоненту $E_{1}$.

Теорема 5. Проекиия $\Pi_{1} \mathcal{A}(\varepsilon)=\mathcal{A}_{1}(\varepsilon), 0 \leqslant \varepsilon \leqslant \varepsilon_{0}$, является равномерным по $\rho \in \mathcal{H}(\sigma)$ аттрактором для прочесса $\mathcal{U}_{\rho}^{\varepsilon}$. Кроме того, семейство аттракторов $\mathcal{A}_{1}(\varepsilon), \varepsilon \in\left[0 ; \varepsilon_{0}\right]$, полунепрерывно сверху в точке $\varepsilon=0$ :

$$
\operatorname{dist}_{E_{1}}\left(\mathcal{A}_{1}(\varepsilon), \mathcal{A}_{1}(0)\right) \rightarrow 0 \quad n p u \quad \varepsilon \rightarrow 0 .
$$

\section{СПИСОК ЦИТИРОВАННОЙ ЛИТЕРАТУРЫ}

1. Вишик М. И., Чепыжев В. В. // УМН. 1995. Т. 50. №4. С. 151. 2. Бабин А. В., Вишик М. И. Аттракторы эволюционньх уравнений. М.: Наука, 1989. 3. Hale J. K. Raugel G. // Annali Mat. Pura Appl. (IV). 1989. V. CLIV. P. 201-326. 4. Hale J. K. Raugel G. // J. Differential Equations. 1988. V. 73. № 2. P. 197-214. 5. Hale J. K. Raugel G. // J. Dynamics Differential Equations. 1990. V. 2. № 1. P. 19-67. 6. Chepyzhov V. V., Vishik M. I. // J. Math. Pures Appl. 1994. V. 73. № 3. Р. 279-333. 7. Лионс ЖК. Л. Некоторые методы решения нелинейных краевых задач. М.: Мир, 1972.

Московский государственный университет им. М. В. Ломоносова 